\title{
roe-solca.ec
}

\section{Condrosarcoma mesenquimal en región pectoral, reporte de caso}

\section{*Correspondencia: \\ rominasolorzano16@gmail.com}

Km 13 vía Samborondón, Urbanización San Antonio Mz 4 SI 12, Guayaquil, Ecuador. Teléfono [593] 0995116563

\section{Conflicto de intereses: Los autores declaran no tener conflictos de intereses.}

Recibido: 4 de Febrero 2021

Aceptado: 21 de Marzo, 2021

Publicado: 30 de Abril, 2021

Editor: Dr. Felipe Campoverde

Membrete bibliográfico:

Ortega J, Solórzano E. Condrosarcoma mesenquimal en región pectoral, reporte de caso. Rev. Oncol. Ecu 2021;31(1):86-92.

ISSN: 2661-6653

DOI: https://doi.org/10.33821/551

Copyright Ortega J, et al. Este artículo es distribuido bajo los términos de Creative Commons Attribution License, el cual permite el uso y redistribución citando la fuente y al autor original.

\section{Mesenchymal chondrosarcoma in the pectoral re- gion, case report}

\section{John Ortega Cedeño1, Evelyn Solórzano Pérez*2}

1. Servicio de medicina Interna, Hospital General del Norte de Guayaquil IESS Los Ceibos.

2. Escuela de Medicina, Facultad de Ciencias Médicas, Universidad Católica Santiago de Guayaquil, Ecuador.

\section{Resumen}

Introducción: El condrosarcoma mesenquimal extraesquelético (CME), corresponde al 1\% de todos los tumores malignos de los tejidos blandos. Se localizan principalmente en la región de la cabeza y cuello, sobre todo en la órbita, y en la duramadre del cráneo, seguida de las extremidades inferiores particularmente a nivel del muslo. La ubicación pectoral es rara, motivo de reporte.

Reporte de caso: Paciente hombre de 38 años quien desarrolla un nódulo de aproximadamente $2 \mathrm{~cm}$ de diámetro localizado en región pectoral derecha con dolor mínimo a la palpación y crecimiento rápido. Dos meses después, al momento la de resección, el nódulo mide $7.5 \times 6.5 \times 3.5 \mathrm{~cm}$, y pesa $106 \mathrm{~g}$. Presenta aspecto lobulado, color café grisáceo, al corte es de consistencia cauchosa y superficie blanquecina nodular con áreas centrales de aspecto mineralizado/calcificado. Se procesa 6 cortes de parafina y se diagnostica como condrosarcoma mesenquimal (CM).

Evolución: El paciente fue egresado y está en control por consulta externa no ha desarrollado recidivas hasta el momento.

Conclusiones: El crecimiento acelerado de una masa de consistencia cartilaginosa se correlacionó en este paciente con la presencia de un condrosarcoma mesenquimal extraesquelético.

Palabras claves:

DeCS: Condrosarcoma Mesenquimal, Neoplasias de los Tejidos Blandos, Informes de casos;

DOI: 10.33821/551 


\begin{abstract}
Introduction: The extraskeletal mesenchymal chondrosarcoma (ESC) corresponds to $1 \%$ of all malignant soft tissue tumors. They are located mainly in the head and neck region, especially in the orbit, and in the dura mater of the skull, followed by the lower extremities, particularly at the level of the thigh. Pectoral location is rare, reason for report.

Case report: 38-year-old male patient who developed a nodule approximately $2 \mathrm{~cm}$ in diameter located in the right pectoral region with minimal pain on palpation and rapid growth. Two months later, at the time of resection, the nodule measures $7.5 \times 6.5 \times 3.5 \mathrm{~cm}$, and weighs $106 \mathrm{~g}$. It has a lobulated appearance, greyish brown color, when cut it is of a rubbery consistency and a nodular whitish surface with central areas of mineralized / calcified appearance. 6 paraffin sections are processed and diagnosed as mesenchymal chondrosarcoma (CM).
\end{abstract}

Evolution: The patient was discharged and is being monitored by an outpatient clinic. He has not developed recurrences to date.

Conclusions: The accelerated growth of a mass of cartilaginous consistency was correlated in this patient with the presence of an extraskeletal mesenchymal chondrosarcoma.

Keywords: MESH: Chondrosarcoma, Mesenchymal; Soft Tissue Neoplasms; Case Reports;

DOI: $10.33821 / 551$

\title{
Introducción
}

El condrosarcoma mesenquimal extraesquelético (CME), descrito por primera vez en 1959 por Lichtenstein y Bernstein, corresponde al 1-2\% de todos los tumores malignos de los tejidos blandos, con patrón bimórfico compuesto por células pequeñas y redondas entremezclado con cartílago bien diferenciado y constituye una variante infrecuente y agresiva del condrosarcoma [1-3]. Se localizan principalmente en la región de la cabeza y cuello, sobre todo en la órbita, y en la duramadre del cráneo, seguida de las extremidades inferiores particularmente a nivel del muslo [4, 5]. El abordaje histopatológico incluye la tinción de inmunohistoquímica con vimentina, CD-99 (Cluster of differentiation 99), BCL-2 (B cell Lymphoma 2), proteína S-100, NSE (neuron specific enolase), CK-20 (citoqueratine-20), desmina, sinaptofisina, cromogranina, HHF-35 (muscle actin monoclonal antibody) [1]. Esta comunicación de caso describe un condrosarcoma mesenquimal en región pectoral derecha.

\section{Caso Clínico}

\section{Descripción del caso}

Paciente masculino de 38 años, sin antecedentes médicos de importancia, que acude por presentar nódulo pectoral de $2 \mathrm{~cm}$ de diámetro aproximadamente de dos meses en región pectoral derecha con dolor mínimo a la palpación y crecimiento rápido. Dos meses después de la valoración inicial el tumor midió $7.5 \times 6.5$ × $3.5 \mathrm{~cm}$ por lo que se decidió la resección quirúrgica. 


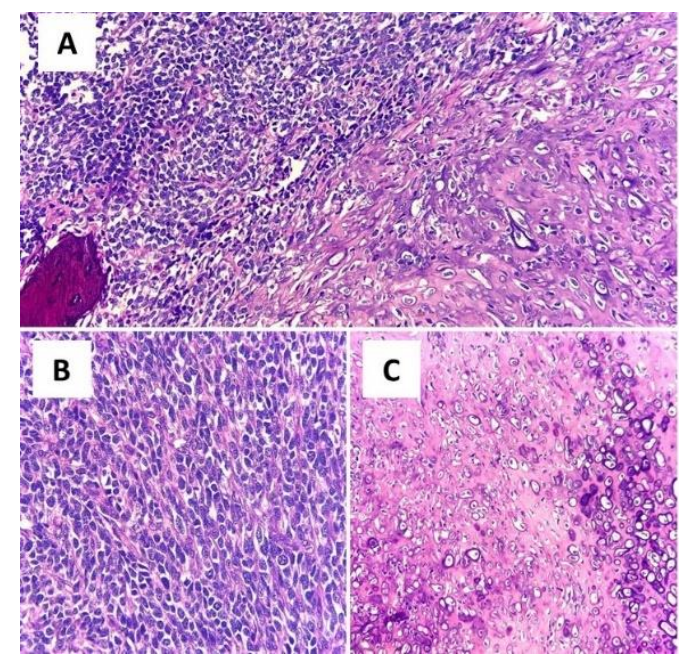

Figura 1 Tinción de hematoxilina y eosina: A) Condrosarcoma mesenquinal (CM) que muestra áreas de células indiferenciadas (izquierda superior) en contraste con su componente condroide, acompañado de calcificación focal (20x). B) componente indiferenciado del CM con células de escaso citoplasma, núcleos redondos y ovoides muy hipercromáticos (40x). C) CM con condroblastos de diferentes tamaños y matriz en diferentes estadios de mineralización (20x)

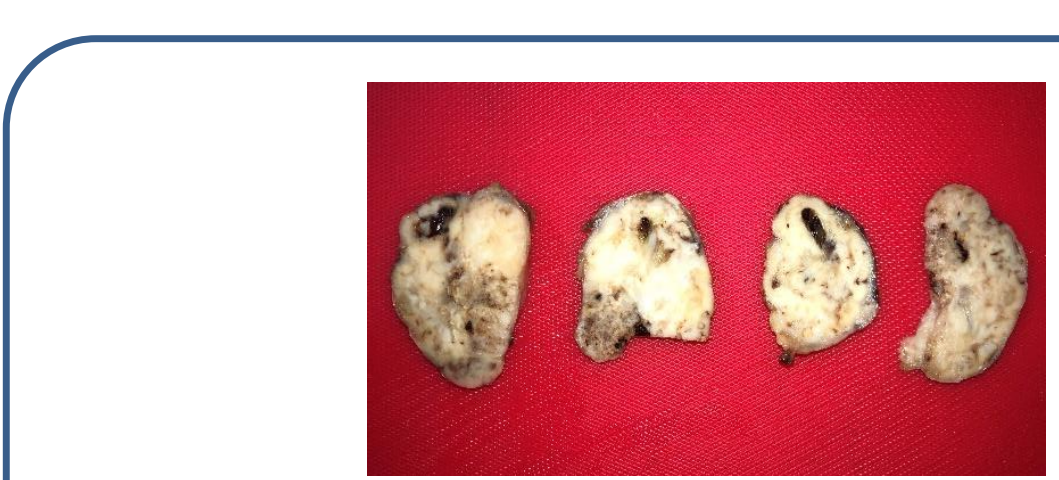

Figura 2 Costes del tumor de consistencia cauchosa y presenta superficie blanquecina nodular.

\section{Taller diagnóstico}

Se realiza en un centro hospitalario resección total del tumor el cual pesó 109 gramos. El diagnóstico histopatológico microscópico muestra una neoplasia de estirpe mesenquimal compuesta por nódulos o sábanas de células de aspecto primitivo, indiferenciadas de citoplasma escaso y núcleos redondos y ovoides con un patrón parcial de empaquetamiento y con vasos de paredes finas comprimidos por dichas células. Se observó un componente importante condroide con atipias marcadas y distintos estadios de mineralización, que llega a formar calcificaciones y osificación parcial, que se fusiona de manera gradual en las áreas indiferenciadas. El tumor también presenta áreas de necrosis abrupta y zonas periféricas de aspecto encapsulado. Se encuentras varias mitosis 1-2 por campo en 20x y 40x. (Figura 1) 


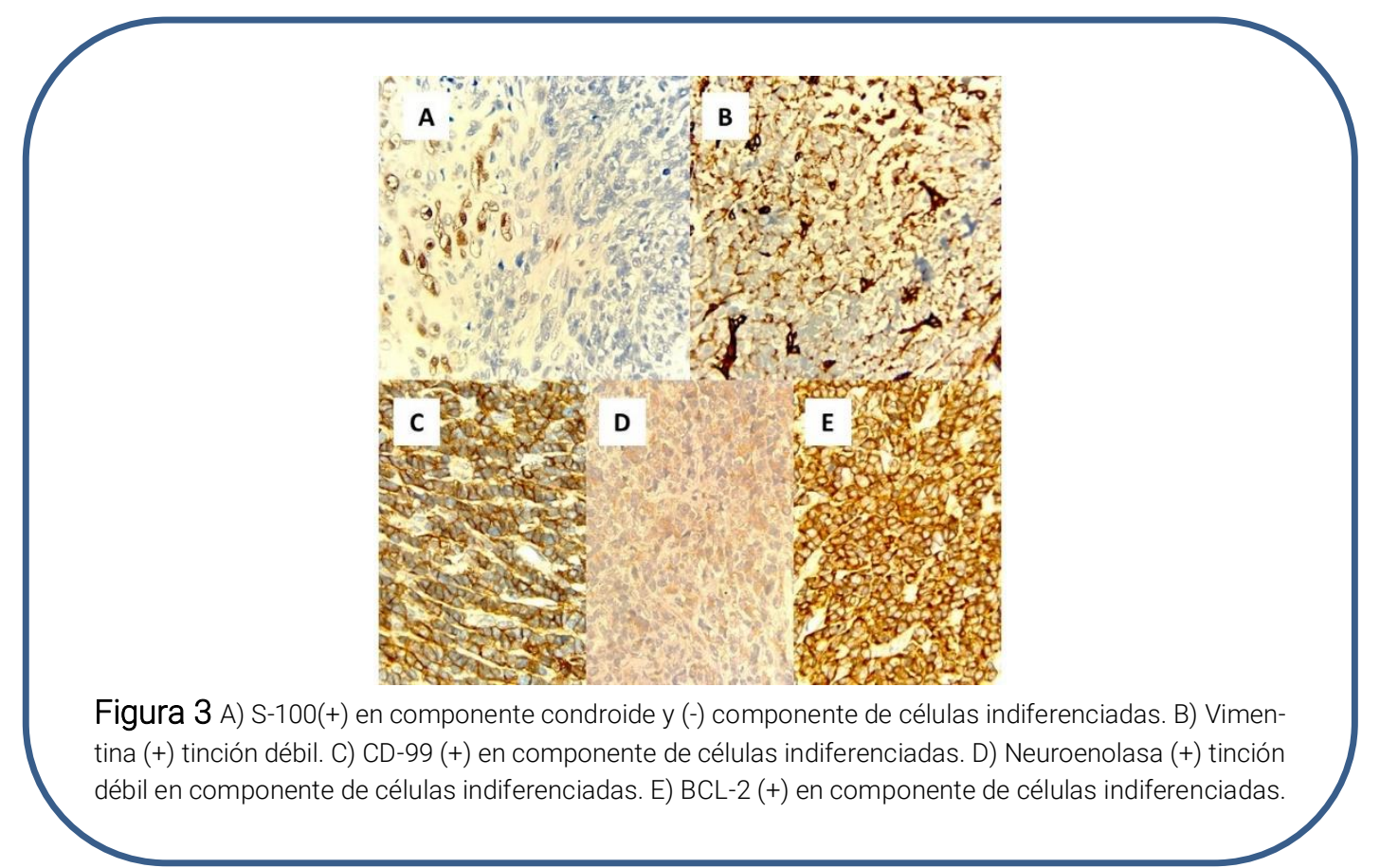

Macroscópicamente, el nódulo se muestra liso, polilobulado, color café grisáceo, pesa 106g y mide $7.5 \times 6.5 \times 3.5 \mathrm{~cm}$. Al corte es de consistencia cauchosa y presenta superficie blanquecina nodular (Figura 2). En región central se encuentran áreas calcificadas y fibrosas. Se procesaron 6 cortes de parafina.

\section{Evolución}

El paciente fue dado de alta luego de la cirugía, se prescribieron analgésicos orales (aines) Acudió al control post operatorio y a una nueva cita para revisión de estudios histopatológicos. Se planteó el manejo expectante sin radio ni quimioterapia. El paciente continúa en valoración de consulta externa sin recidiva del tumor hasta el momento.

\section{Discusión}

En la actualidad se definen varios subtipos de condrosarcomas: intramedular convencional, de células claras, yuxtacortical, mixoide, mesenquimal y mal diferenciado. Los condrosarcomas extraesqueléticos hacen referencia a los tumores cartilaginosos malignos sin compromiso óseo evidente. Aunque la etiología aún no es clara, se han identificado factores predisponentes como: trastornos genéticos, exposición a radiación, agentes químicos, linfedema y antecedentes traumáticos. El condrosarcoma mesenquimal es una variante del condrosarcoma caracterizada microscópicamente por un patrón dimórfico en el que alternan zonas de cartílago bien diferenciado con estroma indiferenciado. El elemento indiferenciado está compuesto de células pequeñas y puede confundirse con un linfoma agresivo, un hemangiopericitoma o un sarcoma de Ewing [5]. La localización más frecuente de la metástasis es el pulmón. Inmunohistoquímicamente, el componente de células pequeñas es positivo para vimentina, CD99 y Leu [6, 7] pero no para la proteína S-100, esta última positividad se encuentra en las áreas condroides como se reporta en este caso. Sin embargo, se piensa que el CM pudiera 
pertenecer a los tumores neuroendócrinos primitivos debido a que muestra positividad para marcadores como proteína S-100 y enolasa neuroespecífica, ambos específicos para tumores de origen neural. El diagnóstico diferencial incluye: condrosarcoma mixoide, hemangiopericitoma, neuroblastoma, rabdomiosarcoma entre otros [3]. Difiere del condrosarcoma convencional en su incidencia, tratándose de un tumor extremadamente raro y muestra un comportamiento más agresivo y una mayor tendencia a afectación de huesos planos y tejidos extra óseos. La posibilidad de recidiva local es del $80 \%$ frente a un $17 \%$ en el condrosarcoma convencional y la supervivencia global es menor de 60\% a los 5 años, a diferencia del convencional que muestra cifras superiores a $85 \%$ para igual período según lo reportado por Cruz y colaboradores [6]. La conducta a seguir es controvertida, aunque la mayoría de los autores plantea que la mejor respuesta está relacionada con el manejo multidisciplinario, siendo la opción más aceptada la combinación de cirugía y radio-quimioterapia coadyuvante [2, 6].El pronóstico del CME es totalmente imprevisible. Huvos reportó un índice de supervivencia a 10 años de $28 \%$, mientras que Nakashima reportó el índice de supervivencia a 5 años de $54.6 \%$ [4].

\section{Conclusiones}

Presentamos el caso de un paciente masculino, en la segunda década de vida con diagnóstico de CM localizado en región pectoral derecho, confirmado histopatológicamente. El CM constituye una enfermedad poco frecuente y por ende es difícil obtener experiencia en su tratamiento en forma aislada. El tratamiento con quimioterapia o radioterapia puede dar resultados favorables aunque no está claramente determinado su impacto en la supervivencia. El crecimiento acelerado de una masa de consistencia cartilaginosa se correlacionó en este paciente con la presencia de un condrosarcoma mesenquimal extraesquelético.

\section{Agradecimientos}

Nota del Editor

La Revista Oncología Ecu

permanece neutral con respecto a los reclamos jurisdiccionales en mapas publicados y afiliaciones institucionales.

\section{Información administrativa}

Abreviaturas

CME: condrosarcoma mesenquimal extraesquelético

\section{Archivos Adicionales}

Ninguno declarado por los autores. 
Fondos

Los autores no recibieron ningún tipo de reconocimiento económico por este trabajo de investigación. Los gastos producidos en la investigación fueron asumidos por los investigadores.

Disponibilidad de datos y materiales

Existe la disponibilidad de datos bajo solicitud al autor de correspondencia. No se reportan otros materiales.

Contribuciones de los autores

Jonathan Darío Ortega Calderón realizó la conceptualización, curación de datos, análisis formal, adquisición de fondos, investigación, recursos, software, redacción - borrador original.

Evelyn Solórzano Pérez realizó la supervisión, validación, visualización, metodología, administración de proyecto, escritura: revisión y edición.

Todos los autores leyeron y aprobaron la versión final del manuscrito.

Aprobación del comité de ética

El protocolo de investigación fue aprobado por el Departamento de Docencia del Hospital Solca - Guayaquil.

Consentimiento para publicación

El presente estudio cuenta con la aprobación y permiso de publicación escrito por parte del paciente.

\section{Referencias}

1. González R, Bologna R, Muñoz R, Mora A. Condrosarcoma mesenquimal extraesquelético en región escapular. A propósito de un caso y revisión de literatura. Revista Española Patología. 2009;42(4):296299. doi: $10.1016 /$ S1699-8855(09)70197-7

2. Bacchetti S, Pegolo E, Adani G, Macrì A, Andreetta C, Ermacora P, Girometti R, Trovò M, Driul L, Di Loreto C, Pasqual E. Extraskeletal myxoid chondrosarcoma: a case report with adjuvant intraoperative treatment. J Surg Case Rep. 2020 Dec 28;2020(12):rjaa503. doi: 10.1093/jscr/rjaa503. PMID: 33391642; PMCID: PMC7769219.

3. Stacchiotti S, Baldi GG, Morosi C, Gronchi A, Maestro R. Extraskeletal Myxoid Chondrosarcoma: State of the Art and Current Research on Biology and Clinical Management. Cancers (Basel). 2020 Sep 21;12(9):2703. doi: 10.3390/cancers12092703. PMID: 32967265; PMCID: PMC7563993.

4. Brenca M, Stacchiotti S, Fassetta K, Sbaraglia M, Janjusevic M, Racanelli D, Polano M, et al. NR4A3 fusion proteins trigger an axon guidance switch that marks the difference between EWSR1 and TAF15 
translocated extraskeletal myxoid chondrosarcomas. J Pathol. 2019 Sep;249(1):90-101. doi: 10.1002/path.5284. Epub 2019 May 14. PMID: 31020999; PMCID: PMC6766969.

5. Wakely PE Jr. Extraskeletal myxoid chondrosarcoma: combining cytopathology with molecular testing to achieve diagnostic accuracy. J Am Soc Cytopathol. 2021 May-Jun;10(3):293-299. doi: 10.1016/j.jasc.2020.07.135. Epub 2020 Jul 31. PMID: $\underline{32828707}$.

6. Cruz O, López F, Fernández P, Nápoles M, Hernández Z, Caballero J, et al . Tratamietno multimodal del condrosarcoma mesenquimal del hueso temporal. Reporte de un caso. Rev Chil Neuro-Psiquiat. 2013 Agosto;3(51):184-190. SCIELO: scielo.cl/S0717.

7. Gallo A, Wilches C, Rivero O, Palau M. Condrosarcoma mesenquimal extraesquelético en un paciente pediátrico: Revisión de la literatura a propósito de un caso. Revista Chilen de Radiología. 2010; 16(3):116-119. SCIELO:scielo.cl/S0717 doi: 10.4067/S0717-93082010000300004. 